Rheumatoid arthritis - comorbidity and clinical aspects.

\section{AB0151 FOLLOW-UP IN A MULTIDISCIPLINARY UNIT IMPROVES PREGNANCY OUTCOME IN INFLAMMATORY ARTHROPATIES ON BIOLOGICAL THERAPY}

O. Ibarguengoitia ${ }^{1}$, I. Calvo ${ }^{1}$, D. Montero ${ }^{2}$, L. Vega ${ }^{2}$, C. García ${ }^{2}$, O. Fernandez ${ }^{2}$, I. Torre ${ }^{2}$, A. R. Inchaurbe ${ }^{2}$, C. E. Perez ${ }^{2}$, J. M. Blanco ${ }^{2}$, E. Cuende ${ }^{2}$, E. Galindez ${ }^{2}$, I. Gorostiza ${ }^{3}$, J. Oraa ${ }^{4}$, M. L. García Vivar', M. E. Ruiz² ${ }^{1}$ Galdakao University Hospital, Rheumatology, Galdakao, Spain; ${ }^{2}$ Basurto University Hospital, Rheumatology, Bilbao, Spain; ${ }^{3}$ Basurto University Hospital, Investigation Unit, Bilbao, Spain; ${ }^{4}$ Basurto University Hospital, Obstetrics and Gynecology, Bilbao, Spain

Background: Women with inflammatory arthropaties have fertility problems and complications during pregnancy and frequently biological therapy (BT) is required for the disease control.

Objectives: To evaluate pregnancy in women with inflammatory arthropaties in a multidisciplinary unit composed of Rheumatologists and Obstetricians: describe disease evolution, complications and treatment used (including BT).

Methods: Retrospective and descriptive study of the evolution of pregnancy in patients with

inflammatory diseases (Rheumatoid Arthritis (RA), Spondyloarthritis (SpA) and Juvenile Idiopathic Arthritis (JIA)) and follow-up in a multidisciplinary unit for more than 15 years (until December 2020). Demographics, maternal disease, time until conception, previous abortions and presence of antibodies were collected. In addition, during follow-up, treatment, abortions, Caesarean sections (C-section), preterm births, disease activity and maternal/fetal complications were collected.

Results: We registered 41 pregnancies (32 women): 20 RA (62.5\%), $9 \mathrm{SpA}$ $(28.1 \%)$ and $3 \mathrm{JIA}(9.4 \%)$. Maternal average age at diagnosis was $27.1 \pm 6.6$ years and average age at childbirth/abortion was $34.9 \pm 5.1$ years.

It took an average time of $9.6 \pm 8.5$ months to conceive. $9.8 \%$ received fertility treatment with in vitro fertilization techniques.

AntiRo antibodies were registered in $7.3 \%$ of patients and $34.1 \%$ had at least 1 antiphospholipid antibody.

At the time of gestational desire/gestation 17 women (12 RA, 4 SpA, 2 JIA) were receiving BT: 7 certolizumab (CZP), 7 adalimumab (ADA), 3 etanercept (ETN). 1 patient was being treated with baricitinib. Due to pregnancy, ADA was changed to CZP in 3 women and BT was stopped in 6 cases ( 3 ETN, 2 ADA, 1 CZP) as well as baricitinib. In 2 cases, ADA was stopped at week 17 of pregnancy (medical indication). Pregnancy was completed with BT (CZP) in 9 cases.

9 abortions were registered prior to follow-up in the unit ( 0.28 abortions/mother) and 3 during follow-up (0.09 abortions/mother): 2 of them in women with CZP. C-section was performed in $26.8 \%$ of cases.

Preterm birth (<37 weeks) happened in $9.7 \%(n: 4)$ of the pregnancies: 1 case in a woman with CZP.

A total of 17 different fetal/maternal complications were registered during follow-up: 6 in the BT group (35.3\%) compared to 11 (64.7\%) in the group without $\mathrm{BT}$, being Intrauterine Growth Restriction (IUGR) more frequent among women with BT. Infections were not more common in patients with BT. Complications are listed in Table 1.

Table 1.

\begin{tabular}{lll}
\hline COMPLICATIONS & WITH BT (n, \%) n: $\mathbf{1 1}$ & WITHOUT BT (n, \%) $\mathbf{n : ~} \mathbf{3 0}$ \\
\hline IUGR & $3(27.3 \%)$ & $1(3.3 \%)$ \\
LOW BIRTH WEIGHT & $2(18.2 \%)$ & $2(6.6 \%)$ \\
INFECTION & $1(9.1 \%)$ & $4(13.3 \%)$ \\
CHOLESTASIS & $0(0 \%)$ & $2(6.6 \%)$ \\
PREECLAMPSIA & $0(0 \%)$ & $1(3.3 \%)$ \\
DIABETES MELLITUS & $0(0 \%)$ & $1(3.3 \%)$ \\
HIGH BLOOD PRESSURE & $0(0 \%)$ & $0(0 \%)$ \\
NEPHROPATY & $0(0 \%)$ & $0(0 \%)$ \\
NEONATAL LUPUS & $0(0 \%)$ & $0(0 \%)$ \\
HEART BLOCK & $(0 \%)$ & $0(0 \%)$ \\
MALFORMATION & $0(0 \%)$ & $0(0 \%)$ \\
HELLP SYNDROME & $0 / 0 \%)$ & $0(0 \%)$ \\
TOTAL & $6(54.6 \%)$ & $11(36.4 \%)$ \\
\hline
\end{tabular}

Regarding concomitant treatment, low dose prednisone was used in $48.8 \%$ of pregnancies, hydroxychloroquine in $51.2 \%$, sulfasalazine in $9.8 \%$ and acetylsalicylic acid in $51.2 \%$. We didn't find differences in the use of these treatments between the two groups.
Median DAS28 among RA patients and available data was under 2.6 throughout pregnancy as well as previously and posteriorly. No differences in median DAS28 were found between women with BT and without BT. SpA patients had BASDAI lower than 4 in both groups during pregnancy and previously.

Conclusion: In our series, as described in the literature, women with inflammatory arthropaties are older and are more likely to have preterm births compared to general population. Fewer abortions were registered during follow-up in the multidisciplinary unit. Appropriate disease control was maintained during pregnancy, also previously and afterwards. We registered more IUGR and low birth weight among women with BT but given the low number of patients with BT no statistically significant conclusions about complications can be drawn. Therefore, more studies among pregnant women with BT are necessary.

Disclosure of Interests: None declared

DOI: 10.1136/annrheumdis-2021-eular.715

\section{\begin{tabular}{|l|l} 
AB0152 & PROSPECTIVE ANALYSIS OF OBSTRUCTIVE SLEEP
\end{tabular} APNEA IN NEWLY DIAGNOSED PATIENTS WITH RHEUMATOID ARTHRITIS, PSORIATIC ARTHRITIS AND PERIPHERAL SPONDYLOARTHRITIS - A SCREENING STUDY}

D. Dejcman ${ }^{1}$, D. Skowasch ${ }^{2}$, C. Pizarro ${ }^{2}$, P. Karakostas ${ }^{1}$, P. Brossart ${ }^{1}$, V. Schäfer ${ }^{1}$. 'University Hospital Bonn, Department of Internal Medicine III Oncology, Hematology, Rheumatology and Stem Cell Transplantation, Bonn, Germany; ${ }^{2}$ University Hospital Bonn, Department of Internal Medicine II Cardiology, Angiology and Pulmonary Care, Bonn, Germany

Background: An increased prevalence of obstructive sleep apnea (OSA) in patients with rheumatoid arthritis (RA), psoriatic arthritis (PSA) and partially in peripheral spondylarthritis ( $\mathrm{pSpA}$ ) has been noted and described for nearly two decades. Until now, there is no study yet on the occurrence of OSA in patients with these entities at the point of first diagnosis. Identifying and treating OSA could prove pivotal in improving cardiovascular risk and quality of life. Furthermore, clinicians require insights into whom to screen.

Objectives: To assess the prevalence of OSA in early RA, PsA and pSpA and strategies for targeted screening.

Methods: We performed a prospective study on patients with first diagnosis of RA, PsA or pSpA, who were screened for day sleepiness (assessed by the Epworth sleepiness scale) and tested for obstructive sleep disorders by out-ofcenter sleep apnea polygraphy (employing Embletta ${ }^{\circledR}$ MPR-PG devices) at the University Hospital of Bonn, Germany. Findings were assessed by three physicians, always including an attending physician responsible for the certified sleep laboratory.

Results: A total of 22 patients with RA (52.38\%), 15 with PsA (35.7\%), and $5(11.9 \%)$ with pSpA were included. Day sleepiness screening was unre markable (ESS <10) in $29(69 \%)$ patients and suspicious for a sleep disorder $(E S S \geq 10)$ in 13 patients $(31 \%)$. Subsequent sleep studies of five patients $(11.9 \%)$ were suspicious for severe sleep apnea $(\mathrm{AHI}>15 / \mathrm{h})$, eight $(19.1 \%)$ for low-to-moderate sleep apnea (AHI 5-15/h) and 29 (69.1\%) were unremarkable $(\mathrm{AHI}<5)$. Of the eight low-to-moderate results, none were symptomatic as in ESS $\geq 10$, resulting in a total of five (11.9\%) guideline-confirming referrals for inpatient polysomnography/ventilation therapy. In comparison with the $\mathrm{PsA} / \mathrm{pSpA}$ group, a diagnosis of RA had no significant relationship with ESS results $(p \approx 0.23)$, nor $A H I$ results $\geq 5 / h \quad(p \approx 0.14)$. The male cohort yielded more pathological sleep studies, but the result was not significant ( $16 \%$ vs. $\sim 5.9 \%$, $p \approx 0.32)$. Higher ESS scores $(\geq 10)$ were not predictive for findings which require a referral $(p \approx 0.37)$. Increased $B M I\left(\geq 25 \mathrm{~kg} / \mathrm{m}^{2}\right)$ was not associated with an $\mathrm{AHI}>5 / \mathrm{h}(\mathrm{p} \approx 0.63)$.

Conclusion: From the point of screening, a number needed to screen of 8.4 (6.25 in the male, 17 in the female group), speaks in favor of overall cost-efficient screening for OSA in newly diagnosed male patients with arthritis diseases. A different study design, comparing early vs. established arthritis cohorts is necessary to assess the role of disease duration in the possible development of OSA REFERENCES:

[1] Chung W-S, Lin C-L. Sleep disorders associated with risk of rheumatoid arthritis. Sleep Breath [Internet]. 2018 Dec 10 [cited 2018 Dec 11];22(4):108391. Available from: http://www.ncbi.nlm.nih.gov/pubmed/29428977.

[2] Vakil M, Park S, Broder A. The complex associations between obstructive sleep apnea and auto-immune disorders: A review. Med Hypotheses [Internet]. 2018;110:138-43. Available from: https://doi.org/10.1016/j. mehy.2017.12.004.

[3] Wali S, Mustafa M, Manzar D, Bawazir Y, Attar S, Fathaldin O, et al. Prevalence of obstructive sleep apnea in patients with rheumatoid arthritis. J Clin Sleep Med. 2020 